\title{
SEMILINEAR INTEGRO-DIFFERENTIAL EQUATIONS WITH COMPACT SEMIGROUPS
}

\author{
D. BAHUGUNA \\ Indian Institute of Technology, Department of Mathematics \\ Kanpur 208016 INDIA \\ S.K. SRIVASTAVA \\ Lucknow University, Department of Mathematics \\ Lucknow 226 007 INDIA
}

(Received July, 1996; Revised May, 1997)

In this paper we study the local and global existence of mild solutions to a class of integro-differential equations in an arbitrary Banach space associated with the operators generating compact semigroups on the Banach space.

Key words: $C_{0}$ Semigroup, Compact Semigroup, Mild Solution.

AMS subject classifications: $34 \mathrm{G} 20,35 \mathrm{~K} 60$.

\section{Introduction}

In this paper we are concerned with the following integro-differential equation considered in a Banach space $X$ :

$$
\begin{gathered}
\frac{d u}{d t}+A u(t)=f(t, u(t))+\int_{t_{0}}^{t} a(t-s) g(s, u(s)) d s, \quad 0 \leq t_{0}<T_{0} \leq \infty \\
u\left(t_{0}\right)=u_{0},
\end{gathered}
$$

where $-A$ is assumed to be an infinitesimal generator of a compact semigroup $T(t)$, $t \geq 0$, on $X$, the nonlinear maps $f, g: J \times U \rightarrow X, J=\left[t_{0}, T_{0}\right), t_{0}<T_{0} \leq \infty$, are continuous where $U$ is an open subset of $X, a \in L^{1}(J)$ and $u_{0}$ is in $U$.

The problem (1.1) for a particular case in which $g=0$ has been considered by Pazy [4], Pavel [3] and others. The existence of a unique mild solution to (1.1) with $g=0$ is assured under the conditions that $-A$ is the infinitesimal generator of a compact semigroup in $X, f(t, u)$ is continuous in both the variables and uniformly locally Lipschitz continuous in $u$. If the Lipschitz continuity of $f$ in $u$ is dropped, then the existence of a mild solution is no more guaranteed. Examples, in which $A=0$ and $f$ is continuous and the differential equations do not have solutions are given in Dieudonne [1] and Yorke [6]. 
Heard and Rankin [2] considered the following integro-differential equation in a Banach space $X$ :

$$
\begin{gathered}
\frac{d u}{d t}+A(t) u(t)=\int_{t_{0}}^{t} a(t, s) g(s, u(s)) d s+f(t, u(t)), \quad t>t_{0} \geq 0 \\
u\left(t_{0}\right)=u_{0}
\end{gathered}
$$

where for each $t \geq 0$, the linear operator $-A(t)$ is the infinitesimal generator of an analytic semigroup in $X$, the nonlinear operator $f$ is defined from $[0, \infty) \times X$ into $X$ and satisfies a Hölder condition of the form

$$
\left\|f\left(t, y_{1}\right)-f\left(t, y_{2}\right)\right\| \leq C\left[|t-s|^{\eta}+\left\|y_{1}-y_{2}\right\|_{\mu}^{\gamma}\right]
$$

$0<\eta, \gamma, \mu<1,\|\|$ is the norm on $X$ and \|\|$_{\mu}$ is the graph norm on $X_{\mu}=$ $D\left(A^{\mu}(0)\right)$, the nonlinear map $g$ is assumed to satisfy a local Lipschitz condition with respect to the norm of $X$ (cf. (A6) in [2]). Also, the uniqueness of solutions is proved under the restriction that the space $X$ is a Hilbert space and $\gamma=1$.

We also consider the global existence of mild solutions to (1.1). Further assumptions are required for global existence of mild solutions as global existence fails quite frequently. We first prove a result related to maximal interval of existence $\left[t_{0}, T_{\text {max }}\right)$ and show that, if $T_{\max }<\infty$, then the solution blows up in a finite time. Then we establish the global existence under certain growth conditions of the maps $f$ and $g$.

\section{Preliminaries}

In this section we mention some relevant notions and collect some results associated with the following initial value problem considered in a Banach space $X$ :

$$
\begin{gathered}
\frac{d u}{d t}+A u(t)=f(t, u(t)), 0<t_{0}<t<T_{0} \leq \infty, \\
u\left(t_{0}\right)=u_{0}
\end{gathered}
$$

where $-A$ is the infinitesimal generator of a compact semigroup $T(t), t \geq 0$ and $f$ is continuous from $J \times U$ into $X, J=\left[t_{0}, T_{0}\right), t_{0}<T_{0} \leq \infty, U$ is an open subset of $X$ and $u_{0}$ is in $U$.

Let $X$ be a Banach space. A one parameter family $T(t), 0 \leq t<\infty$, of bounded linear operators from $X$ into $X$ is called a semigroup of bounded linear operators on $X$ if $(i) T(0)=I, I$ is the identity operator on $X$ and $(i i) \quad T(t+s)=T(t) T(s)$ for every $t, s \geq 0$. The linear operator $A$ defined by

$$
D(A)=\left\{x \in X: \lim _{t \downarrow 0} \frac{T(t) x-x}{t} \text { exists }\right\}
$$

and

$$
A x=\lim _{t \downarrow 0} \frac{T(t) x-x}{t} \text { for } x \in D(A),
$$

is called the infinitesimal generator of the semigroup $T(t)$. Here $D(A)$ denotes the domain of $A$. A semigroup $T(t)$ is called uniformly continuous if 


$$
\lim _{t \downarrow 0}\|T(t)-I\|=0 .
$$

A semigroup $T(t), 0 \leq t<\infty$, of bounded linear operators on $X$ is called a strongly continuous semigroup of bounded linear operators if

$$
\lim _{t \downarrow 0} T(t) x=x \text { for every } x \in X .
$$

A strongly continuous semigroup $T(t)$ is also called as a $C_{0}$ semigroup. A $C_{0}$ semigroup $T(t)$ is called compact for $t>t_{0}$ if for every $t>t_{0}, T(t)$ is a compact operator. $T(t)$ is called compact if it is compact for $t>0$. We note that, if $T(t)$ is compact for $t \geq 0$, then in particular the identity operator is compact and therefore $X$ in this case is finite dimensional. Also, if $T\left(t_{0}\right)$ is compact for some $t_{0}>0$, then $T(t)$ is compact for every $t \geq t_{0}$ since $T(t)=T\left(t-t_{0}\right) T\left(t_{0}\right)$ and $T\left(t-t_{0}\right)$ is bounded.

We shall use the following result on the compact semigroups.

Theorem 2.1: Let $T(t)$ be a $C_{0}$ semigroup. If $T(t)$ is compact for $t>t_{0}$, then $T(t)$ is uniformly continuous for $t>t_{0}$.

We have the following characterization of a compact semigroup in terms of the resolvent operators $R(\lambda: A)$ of its generator $A$.

Theorem 2.2: Let $T(t)$ be a $C_{0}$ semigroup and let $A$ be its infinitesimal generator. $T(t)$ is a compact semigroup if and only if $T(t)$ is uniformly continuous for $t>0$ and $R(\lambda: A)$ is compact for $\lambda \in \rho(A)$.

By a mild solution to (1.1) on $J$ we mean a function $u \in C(J: X)$ satisfying the integral equation

$$
u(t)=T\left(t-t_{0}\right) u_{0}+\int_{t_{0}}^{t} T(t-s)\left[f(s, u(s))+\int_{t_{0}}^{s} a(s-\tau) g(\tau, u(\tau)) d \tau\right] d s .
$$

For the problem $(2.1)$ we have the following existence theorem due to Pazy $[4,5]$.

Theorem 2.3: Let $X$ be Banach space and $U$ be an open subset of $X$. Let $-A$ be the infinitesimal generator of a compact semigroup $T(t), t \geq 0$. If $f: J \times U \rightarrow X$ is continuous then for every $u_{0}$ in $U$, there exists a $t_{1}, t_{0}<t_{1}<T_{0}$, such that (2.1) has a mild solution $\dot{u}$ on $J_{0}=\left[t_{0}, t_{1}\right)$.

The following result is due to Pavel [3] which extends the results of Theorem 2.3.

Theorem 2.4: Suppose that $D$ is a locally closed subset of $X, f: J \times D \rightarrow X$ is continuous where $J=\left[t_{0}, T_{0}\right)$, and the $C_{0}$ semigroup $T(t), t \geq 0$ is compact for $t>0$. A necessary and sufficient condition for the existence of a local mild solution $u:\left[t_{0}, T\left(t_{0}, u_{0}\right)\right) \rightarrow D, t_{0}<T\left(t_{0}, u_{0}\right)<T_{0}$ to $(2.1)$ for $u_{0} \in D$ is

$$
\lim _{h \rightarrow 0} h^{-1} \operatorname{dist}(S(h) z+h f(t, z): D)=0
$$

for all $t \in\left[t_{0}, T_{0}\right)$ and $z \in D$.

\section{Local Existence}

Our aim is to extend the results of Theorem 2.3 to the initial value problem (1.1). Below we state and prove the following existence result for (1.1).

Theorem 3.1: Let $X$ be a Banach space, $U$ be an open subset of $X$ and $J=$ $\left[t_{0}, T_{0}\right), t_{0}<T_{0} \leq \infty$. Let $-A$ be the infinitesimal generator of a compact semi- 
group $T(t), t \geq 0$. If the nonlinear maps $f, g: J \times U \rightarrow X$ are continuous and a is locally integrable in $J$, then for every $u_{0} \in X$ there exists a $t_{1}, t_{0}<t_{1}<T_{0}$, such that (2.1) has a mild solution $u$ on $\left[t_{0}, t_{1}\right)$. that

Proof: Let $T$ be such that $t_{0}<T<T_{0} \leq \infty$. Let $M$ be a positive constant such

$$
\|T(t)\| \leq M \text { for } 0 \leq t \leq T
$$

Let $\rho>0$ be such that

$$
B_{\rho}\left(u_{0}\right)=\left\{v \in X:\left\|v-u_{0}\right\| \leq \rho\right\} \subset U .
$$

Choose $t^{\prime}>t_{0}$ such that

$$
\begin{gathered}
\|f(t, v)\| \leq N_{1} \\
\|g(t, v)\| \leq N_{2}
\end{gathered}
$$

for $t_{0} \leq t \leq t^{\prime}, v \in B_{\rho}\left(u_{0}\right)$ with positive constants $N_{1}$ and $N_{2}$. Again choose $t^{\prime \prime}>t_{0}$ such that

$$
\left\|T\left(t-t_{0}\right) u_{0}-u_{0}\right\|<\frac{\rho}{2} \text { for } t_{0} \leq t \leq t^{\prime \prime}
$$

Let

where $a_{T}=\int_{t_{0}}^{T}|a(s)| d s$. Now we set

$$
t_{1}=\min \left\{T, t^{\prime}, t^{\prime \prime}, t_{0}+\frac{\rho}{2 M\left(N_{1}+a_{T} N_{2}\right)}\right\}
$$

$$
Y=C\left(\left[t_{0}, t_{1}\right]: X\right)
$$

and

$$
S=\left\{u \in Y: u\left(t_{0}\right)=u_{0}, u(t) \in B_{\rho}\left(u_{0}\right) \text { for } t_{0} \leq t \leq t_{1}\right\}
$$

We note that $S$ is a bounded, closed and convex subset of $Y$. We define a map $F: S \rightarrow Y$ given by

$(F u)(t)=T\left(t-t_{0}\right) u_{0}+\int_{t_{0}}^{t} T(t-s)\left[f(s, u(s))+\int_{t_{0}}^{s} a(s-\tau) g(\tau, u(\tau)) d \tau\right] d s$.

For $u \in S$, we have

$$
\begin{gathered}
\left\|(F u)(t)-u_{0}\right\| \leq\left\|T\left(t-t_{0}\right) u_{0}-u_{0}\right\| \\
+\left\|\int_{t_{0}}^{t} T(t-s)\left[f(s, u(s))+\int_{t_{0}}^{s} a(s-\tau) g(\tau, u(\tau)) d \tau\right] d s\right\| \\
\leq \frac{\rho}{2}+\left(t_{1}-t_{0}\right) M\left(N_{1}+a_{T} N_{2}\right)
\end{gathered}
$$


$\leq \rho$

Thus $F: S \rightarrow S$. Now we show that $F$ is continuous from $S$ into $S$. To show this, we first observe that since $f$ and $g$ are continuous in $\left[t_{0}, T\right] \times U$, it follows that any $\epsilon>0$ and for a fixed $u \in B_{\rho}\left(u_{0}\right)$ there exist $\delta_{1}(u), \delta_{2}(u)>0$ such that for any $v \in B_{\rho}\left(u_{0}\right)$, we have

$$
\|u-v\|_{Y} \leq \delta_{1}(u) \Rightarrow\|f(t, u(t))-f(t, v(t))\| \leq \frac{\epsilon}{2 T M}
$$

and

$$
\|u-v\|_{Y} \leq \delta_{2}(u) \Rightarrow\|g(t, u(t))-g(t, v(t))\| \leq \frac{\epsilon}{2 a_{T} T M}
$$

Let

$$
\delta(u)=\min \left\{\delta_{1}(u), \delta_{2}(u)\right\}
$$

Then for any $v \in S,\|u-v\|_{Y}<\delta(u)$ implies that

$$
\begin{aligned}
& \|(F u)(t)-(F v)(t)\| \leq \int_{t_{0}}^{t}\|T(t-s)\|\|f(s, u(s))-f(s, v(s))\| d s \\
& +\int_{t_{0}}^{t}\|T(t-s)\|\left(\int_{t_{0}}^{s}|a(s-\tau)|\|g(\tau, u(\tau))-g(\tau, v(\tau))\| d \tau\right) d s .
\end{aligned}
$$

Thus, $F: S \rightarrow S$ is continuous. Let

$$
\widetilde{S}=F(S)
$$

and for fixed $t \in\left[t_{0}, t_{1}\right]$, let

$$
S(t)=\{(F u)(t): u \in S\}
$$

Since $S\left(t_{0}\right)=\left\{u_{0}\right\}, S\left(t_{0}\right)$ is precompact in $X$. For $t>t_{0}$ and $0<\epsilon<t-t_{0}$, let

$$
\begin{gathered}
\left(F_{\epsilon} u\right)(t)=T\left(t-t_{0}\right) u_{0}+\int_{t_{0}}^{t-\epsilon} T(t-s)\left[f(s, u(s))+\int_{t_{0}}^{s} a(s-\tau) g(\tau, u(\tau)) d \tau\right] d s \\
=T\left(t-t_{0}\right) u_{0}+T(\epsilon) \int_{t_{0}}^{t-\epsilon} T(t-s-\epsilon)[f(s, u(s)) \\
\left.+\int_{t_{0}}^{s} a(s-\tau) g(\tau, u(\tau)) d \tau\right] d s
\end{gathered}
$$


The compactness of the semigroup $T(t)$ for every $t>0$ and (3.3) imply that for every $\epsilon, 0<\epsilon<t-t_{0}$, the set

$$
S_{\epsilon}(t)=\left\{\left(F_{\epsilon} u\right)(t): u \in S\right\}
$$

is precompact in $X$. Now, for any $u \in S$, we have

$$
\begin{gathered}
\left.\left\|(F u)(t)-\left(F_{\epsilon} u\right)(t)\right\| \leq \int_{t-\epsilon}^{t} \| T(t-s)[f s, u(s))+\int_{t_{0}}^{s} a(s-\tau) g(\tau, u(\tau)) d \tau\right] \| d s \\
\leq \epsilon M\left(N_{1}+a_{T} N_{2}\right) .
\end{gathered}
$$

From (3.4) it follows that the set $S(t)$ is precompact. Now we show that $\widetilde{S}$ is equicontinuous. For $r_{1}, r_{2} \in\left[t_{0}, t_{1}\right]$ with $r_{1}<r_{2}$, we have

$$
\begin{gathered}
\left\|(F u)\left(r_{2}\right)-(F u)\left(r_{1}\right)\right\| \leq\left\|\left(T\left(r_{2}-t_{0}\right)-T\left(r_{1}-t_{0}\right)\right) u_{0}\right\| \\
\left(N_{1}+a_{T} N_{2}\right) \int_{t_{0}}^{r_{1}}\left\|T\left(r_{2}-s\right)-T\left(r_{1}-s\right)\right\| d s \\
+\left(r_{2}-r_{1}\right) M\left(N_{1}+a_{T} N_{2}\right) .
\end{gathered}
$$

Since $T(t)$ is compact, Theorem 2.1 implies that $T(t)$ is continuous in the uniform operator topology for $t>0$. Therefore, the right-hand side of (3.5) tends to zero as $r_{2}-r_{1}$ tends to zero. Thus $\widetilde{S}$ is equicontinuous. Also, $\widetilde{S} \underset{\sim}{\text { is bounded. It follows }}$ from the Arzela-Ascoli theorem (cf. see Dieudonne [1]), that $\widetilde{S}$ is precompact. The existence of a fixed point of $F$ in $S$ is a consequence of Schauder's fixed point theorem and any fixed point of $F$ in $S$ is a mild solution to (1.1) on $\left[t_{0}, t_{1}\right)$.

\section{Global Existence}

In this section we consider the global existence of mild solution to (1.1). For (2.1) we have the following result.

Theorem 4.1: Suppose $-A$ is the infinitesimal generator of a compact semigroup $T(t), t>0$ on $X$. If $f:\left[t_{0}, \infty\right) \times X \rightarrow X$ is continuous and maps bounded subsets of $\left[t_{0}, \infty\right) \times X$ into bounded subsets in $X$, then for every $u_{0} \in X$ the equation (2.1) has a mild solution $u$ on a maximal interval of existence $\left[t_{0}, T_{\max }\right)$ and, if $T_{\text {max }}<\infty$, then

$$
\lim _{T \uparrow T_{\max }}\|u(t)\|=\infty
$$

In the following theorem we extend the results of Theorem 4.1 to the problem (1.1).

Theorem 4.2: Suppose $-A$ is the infinitesimal generator of a compact semigroup $T(t), t>0$ on $X$. If $f, g:\left[t_{0}, \infty\right) \times X \rightarrow X$ are continuous and map bounded subsets of $\left[t_{0}, \infty\right) \times X$ into bounded subsets in $X$ and a is locally integrable in $\left[t_{0}, \infty\right)$, 
then for every $u_{0} \in X$ the equation (1.1) has a mild solution $u$ on a maximal interval of existence $\left[t_{0}, T_{\text {max }}\right)$ and, if $T_{\text {max }}<\infty$, then

$$
\lim _{t \uparrow T_{\max }}\|u(t)\|=\infty .
$$

Proof: From Theorem 3.1 we have the existence of a local mild solution $u \in$ $C\left(\left[t_{0}, t_{1}\right): X\right)$ for some $t_{0}<t_{1}$ to $(1.1)$ given by

$$
u(t)=T\left(t-t_{0}\right) u_{0}+\int_{t_{0}}^{t}\left[f(s, u(s))+\int_{t_{0}}^{s} a(s-\tau) g(\tau, u(\tau)) d \tau\right] d s .
$$

Suppose that $u\left(t_{1}\right)<\infty$. Consider the problem

$$
\begin{gathered}
\frac{d v}{d t}+A v(t)=f(t, v(t))+\int_{t_{1}}^{t} a(t-s) g(s, v(s 0) d s \\
v\left(t_{1}\right)=u\left(t_{1}\right) .
\end{gathered}
$$

From Theorem 3.1 we have that there exists a mild solution $v \in C\left(\left[t_{1}, t_{2}\right): X\right)$ for some $t_{2}, t_{1}<t_{2}<\infty$ to (4.1) given by

$$
\begin{aligned}
& v(t)=T\left(t-t_{1}\right) u\left(t_{1}\right)+\int_{t_{1}}^{t} T(t-s)\left[f(s, v(s))+\int_{t_{1}}^{s} a(s-\tau) g(\tau, v(\tau)) d \tau\right] d s \\
& =T\left(t-t_{1}\right)\left[T\left(t_{1}-t_{0}\right) u_{0}+\int_{t_{0}}^{t_{1}} T\left(t_{1}-s\right)\left[f(s, u(s))+\int_{t_{0}}^{s} a(s-\tau) g(\tau, u(\tau)) d \tau\right] d s\right] \\
& +\int_{t_{1}}^{t} T(t-s)\left[f(s, v(s))+\int_{t_{1}}^{s} a(s-\tau) g(\tau, v(\tau)) d \tau\right] d s \\
& =T\left(t-t_{0}\right) u_{0}+\int_{t_{0}}^{t_{1}} T(t-s)\left[f(s, u(s))+\int_{t_{0}}^{s} a(s-\tau) g(\tau, u(\tau)) d \tau\right] d s \\
& +\int_{t_{1}}^{t} T(t-s)\left[f(s, v(s))+\int_{t_{1}}^{s} a(s-\tau) g(\tau, v(\tau)) d \tau\right] d s .
\end{aligned}
$$

We define $\tilde{u}:\left[t_{0}, t_{2}\right) \rightarrow X$ by

$$
\tilde{u}(t)= \begin{cases}u(t), & t \in\left[t_{0}, t_{1}\right), \\ v(t), & t \in\left[t_{1}, t_{2}\right) .\end{cases}
$$


Then $\tilde{u} \in C\left(\left[t_{0}, t_{2}\right): X\right)$ and for $t_{1}<t<t_{2}$, we have

$$
\begin{gathered}
\tilde{u}(t)=T\left(t-t_{0}\right) u_{0}+\int_{t_{0}}^{t_{1}} T(t-s)\left[f(s, \tilde{u}(s))+\int_{t_{0}}^{s} a(s-\tau) g(\tau, \tilde{u}(\tau)) d \tau\right] d s \\
+\int_{t_{1}}^{t} T(t-s)\left[f(s, \tilde{u}(s))+\int_{t_{1}}^{s} a(s-\tau) g(\tau, \tilde{u}(\tau)) d \tau\right] d s \\
=T\left(t-t_{0}\right) u_{0}+\int_{t_{0}}^{t} T(t-s) f(s, \tilde{u}(s)) d s \\
+\int_{t_{0}}^{t_{1}} \int_{t_{0}}^{s} T(t-s) a(s-\tau) g(\tau, \tilde{u}(\tau)) d \tau d s \\
+\int_{t_{1}}^{t} \int_{t_{1}}^{s} T(t-s) a(s-\tau) g(\tau, \tilde{u}(\tau)) d \tau d s .
\end{gathered}
$$

Changing the order of integration in (4.2), we get

$$
\begin{gathered}
\tilde{u}(t)=T\left(t-t_{0}\right) u_{0}+\int_{t_{0}}^{t} T(t-s) f(s, \tilde{u}(s)) d s \\
+\int_{t_{0}}^{t_{1}} \int_{r}^{t_{1}} T(t-s) a(s-\tau) g(\tau, \tilde{u}(\tau)) d s d \tau \\
+\int_{t_{1}}^{t} \int_{r}^{t} T(t-s) a(s-\tau) g(\tau, \tilde{u}(\tau)) d s d \tau \\
T\left(t-t_{0}\right) u_{0}+\int_{t_{0}}^{t} T(t-s)\left[f(s, \tilde{u}(s))+\int_{t_{0}}^{s} a(s-\tau) g(\tau, \tilde{u}(\tau)) d \tau\right] d s
\end{gathered}
$$

From (4.3) we have that $\tilde{u}$ is a mild solution to (1.1) on $\left[t_{0}, t_{2}\right)$. Now, suppose that $\left[t_{0}, T_{\text {max }}\right)$ is the maximal interval to which the solution $u$ of (1.1) can be extended. If $T_{\text {max }}<\infty$, then we show that $\|u(t)\| \rightarrow \infty$ as $t \uparrow T_{\text {max }}$. It suffices to show that $\varlimsup_{\lim } T_{\text {max }}\|u(t)\|-\infty$. If $\varlimsup_{\lim }{ }_{t \uparrow T_{\text {max }}}\|u(t)\|<\infty$, then there exists a sequence $t_{n} \uparrow T_{\max }$ such that $\left\|u\left(t_{n}\right)\right\| \leq K$ for some constant $K$ and for all $n$. Suppose that $\|T(t)\| \leq M$ for $t \leq t \leq T_{\max }$ and let

$$
N_{1}=\sup \left\{\|f(t, v)\|: t_{0} \leq t \leq T_{\text {max }}, \| v \mid \leq M(K+1)\right\}
$$


and

$$
N_{2}=\sup \left\{\|g(t, v)\|: t_{0} \leq t \leq T_{\max },\|v\| \leq M(K+1)\right\}
$$

Using the continuity of $u$ and the assumption that $\varlimsup_{\lim }\|u(t)\|<\infty$, we can find a sequence $\left\{h_{n}\right\}$ such that $h_{n} \rightarrow 0,\|u(t)\| \leq M(K+1)$ for $t_{n} \leq t \leq t_{n}+h_{n}$ and $\left\|u\left(t_{n}+h_{n}\right)\right\|=M(K+1)$. But then we have

$$
\begin{gathered}
M(K+1)=\left\|T\left(t_{n}+h_{n}\right)\right\| \\
\leq\left\|T\left(h_{n}\right) u\left(t_{n}\right)\right\| \\
+\int_{t_{n}}^{t_{n}+h_{n}}\left\|T\left(t_{n}+h_{n}-s\right)\left[f(s, u(s))+\int_{t_{n}}^{s} a(s-\tau) g(\tau, u(\tau)) d \tau\right]\right\| d s \\
\leq M K+h_{n}\left(N_{1}+a_{T_{\text {max }}} N_{2}\right) M
\end{gathered}
$$

which gives a contradiction as $h_{n} \rightarrow 0$. Hence,

$$
\varlimsup_{t \uparrow T_{\text {max }}}\|u(t)\|=\infty \text {. }
$$

This completes the proof.

Finally, we prove the following global existence result for (1.1).

Theorem 4.3: Let $-A$ be the infinitesimal generator of a compact semigroup, $T(t), t \geq 0$ on $X$. Let $f, g:\left[t_{0}, \infty\right) \times X \rightarrow X$ be continuous functions mapping bounded subsets $\left[t_{0}, \infty\right) \times X$ into bounded subsets of $X$ and a be locally integrable in $\left[t_{0}, \infty\right)$, then any one of the following two conditions is sufficient for the global existence of a mild solution $u$ to (1.1):

(i) There exist a continuous function $k_{0}:\left[t_{0}, \infty\right) \rightarrow[0, \infty)$ such that $\|u(t)\| \leq$ $k_{0}(t)$ for every $t$ in the interval of existence of $u$.

(ii) There exist functions $k_{i}:\left[t_{0}, \infty\right) \rightarrow[0, \infty), i=1,2,3,4$; such that $k_{1}, k_{2}, a * k_{3}$, and $a * k_{4}$ are locally integrable on $\left[t_{0}, \infty\right)$, and for $t_{0} \leq t<\infty, v \in X$

$$
\begin{aligned}
& \|f(t, v)\| \leq k_{1}(t)\|v\|+k_{2}(t), \\
& \|g(t, v)\| \leq k_{3}(t)\|v\|+k_{4}(t)
\end{aligned}
$$

where

for $i=3,4$.

$$
a * k_{i}(t)=\int_{t_{0}}^{t} a(t-s) k_{i}(s) d s
$$

Proof: $(i)$ Since for any $t_{1}, t_{0}<t_{1}<\infty\left\|u\left(t_{1}\right)\right\| \leq k_{0}\left(t_{1}\right)<\infty$, from Theorem 4.2 , it follows that the solution $u$ can be extended beyond $t_{1}$, hence the solution $u$ exists globally.

(ii) The mild solution $u$ to (1.1) is given by 


$$
u(t)=T\left(t-t_{0}\right) u_{0}+\int_{t_{0}}^{t} T(t-s)\left[f(s, u(s))+\int_{t_{0}}^{s} a(s-\tau) g(\tau, u(\tau)) d \tau\right] d s .
$$

Let $\|T(t)\| \leq M e^{\omega t}$. Multiplying the above equation by $e^{-\omega\left(t-t_{0}\right)}$ and taking the norm, we have

$$
\begin{gathered}
e^{-\omega\left(t-t_{0}\right)}\|u(t)\| \leq M\left\|u_{0}\right\|+M \int_{t_{0}}^{t} e^{-\omega\left(s-t_{0}\right)}[\|f(s, u(s))\| \\
\left.+\int_{t_{0}}^{s}|a(s-\tau)|\|g(\tau, u(\tau))\| d \tau\right] d s
\end{gathered}
$$

For $t \in\left[t_{0}, \infty\right)$, set

$$
\xi(t)=M\left\|u_{0}\right\|+M \int_{t_{0}}^{t} e^{-\omega\left(s-t_{0}\right)}\left[k_{2}(s)+\int_{t_{0}}^{s}|a(s-\tau)| k_{4}(\tau) d \tau\right] d s .
$$

From (4.6) we have

$$
\begin{aligned}
& e^{-\omega\left(t-t_{0}\right)}\|u(t)\| \\
& \quad \leq \xi(t)+M \int_{t_{0}}^{t} e^{-\omega\left(s-t_{0}\right)}\left[k_{1}(s)\|u(s)\|+\int_{t_{0}}^{s}|a(s-\tau)| k_{3}(s) \| u(\tau \| d \tau] d s .\right.
\end{aligned}
$$

For $t_{0} \leq r \leq t$, we have

$$
\begin{gathered}
e^{-\omega\left(t-t_{0}\right)}\|u(r)\| \\
\leq \xi(r)+M \int_{t_{0}}^{r} e^{-\omega\left(s-t_{0}\right)}\left[k_{1}(s)\|u(s)\|+\int_{t_{0}}^{s}|a(s-\tau)| k_{3}(s) \| u(\tau \| d \tau] d s\right. \\
\leq \xi(r)+M \int_{t_{0}}^{r} e^{-\omega\left(s-t_{0}\right)_{\left[k_{1}\right.}(s)} \\
\left.+\int_{t_{0}}^{s}|a(s-\tau)| k_{3}(s)\right] \sup _{t_{0} \leq \tau \leq s}\|u(\tau)\| d s .
\end{gathered}
$$

Taking the supremum over $\left[t_{0}, t\right]$ on both the sides of $(4.7)$, we get

$$
\begin{gathered}
e^{-\omega\left(t-t_{0}\right)} \sup _{t_{0} \leq r<t}\|u(r)\| \leq \sup _{t_{0} \leq r \leq t} \xi(r)+M \int_{t_{0}}^{t} e^{-\omega\left(s-t_{0}\right)}\left[k_{1}(s)\right. \\
\left.+\int_{t_{0}}^{s}|a(s-\tau)| k_{3}(\tau) d \tau\right] \sup _{t_{0} \leq r \leq s}\|u(\tau)\| d s
\end{gathered}
$$


Gronwall's inequality implies that

$$
\begin{aligned}
& e^{-\omega\left(t-t_{0}\right)} \sup _{t_{0} \leq r \leq t}\|u(r)\| \\
& \leq \sup _{t_{0} \leq r \leq t} \xi(r)+M \int_{t_{0}}^{t}\left[e ^ { - \omega ( s - t _ { 0 } ) } [ k _ { 1 } ( s ) + \int _ { t _ { 0 } } ^ { s } | a ( s - \tau ) | k _ { 3 } ( \tau ) d \tau ] \operatorname { e x p } \left\{\int _ { s } ^ { t } \left[k_{1}(u)\right.\right.\right. \\
&\left.\left.\left.+\int_{t_{0}}^{s}|a(u-\tau)| k_{3}(\tau) d \tau\right] d u\right\}\right] \sup _{t_{0} \leq r \leq s} \xi(\tau) d s .
\end{aligned}
$$

Inequality (4.9) implies that $\|u(t)\|$ is bounded by a continuous function and from (i) we get the global existence of the mild solution $u$ to (1.1). This completes the proof.

\section{Acknowledgements}

The authors would like to thank the referee for his valuable suggestions for improving the original manuscript.

\section{References}

[1] Dieudonne, J., Foundation of Modern Analysis, Academic Press, New York 1960.

[2] Heard, M.L. and Rankin, S.M., A semilinear parabolic integro-differential equation, J. Diff. Eqns. 71 (1988), 201-233.

[3] Pavel, N.H., Invariant sets for a class of semilinear equations of evolution, Nonlinear Anal. 1 (1977), 187-196.

[4] Pazy, A., A class of semilinear equations of evolution, Israel J. Math. 20 (1975), 23-36.

[5] Pazy, A., Semigroups of Linear Operators and Applications to Partial Differential Equations, Springer-Verlag, New York 1983.

[6] Yorke, J., A continuous differential equation in Hilbert space without existence, Funk. Ekvaciaj 12 (1970), 19-21. 


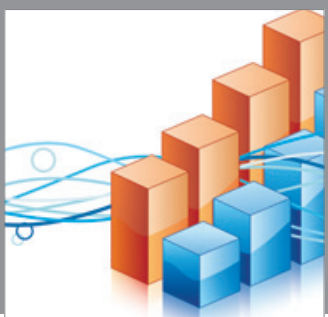

Advances in

Operations Research

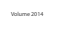

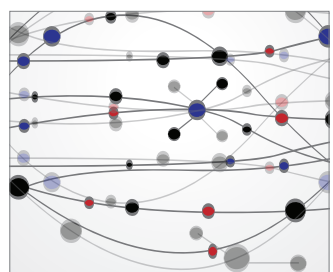

\section{The Scientific} World Journal
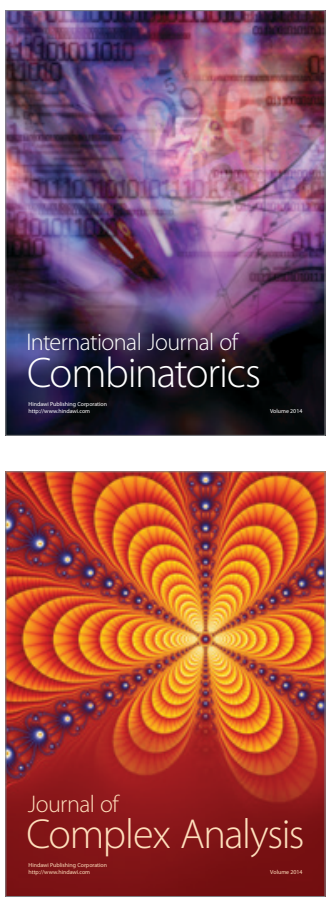

International Journal of

Mathematics and

Mathematical

Sciences
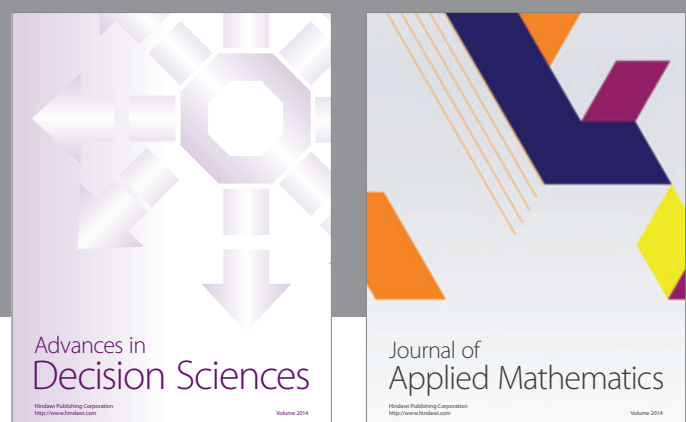

Journal of

Applied Mathematics
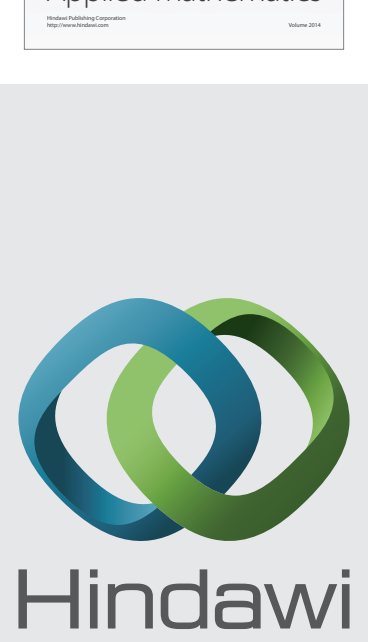

Submit your manuscripts at http://www.hindawi.com
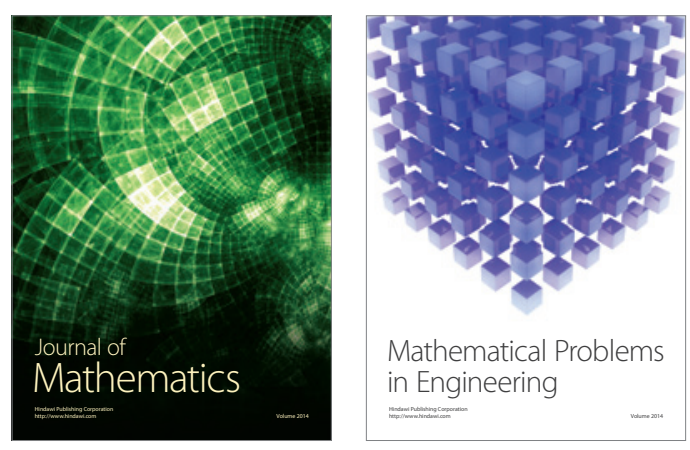

Mathematical Problems in Engineering
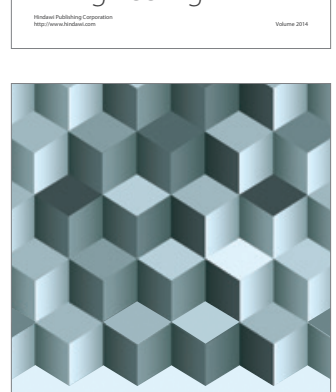

Journal of

Function Spaces
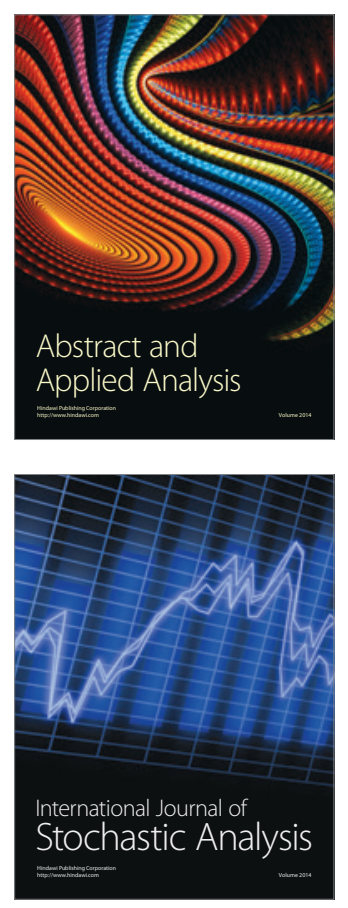

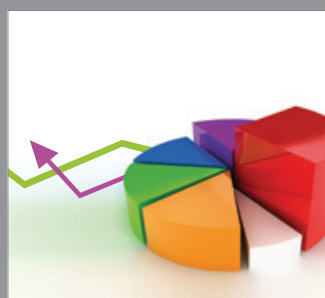

ournal of

Probability and Statistics

Promensencen
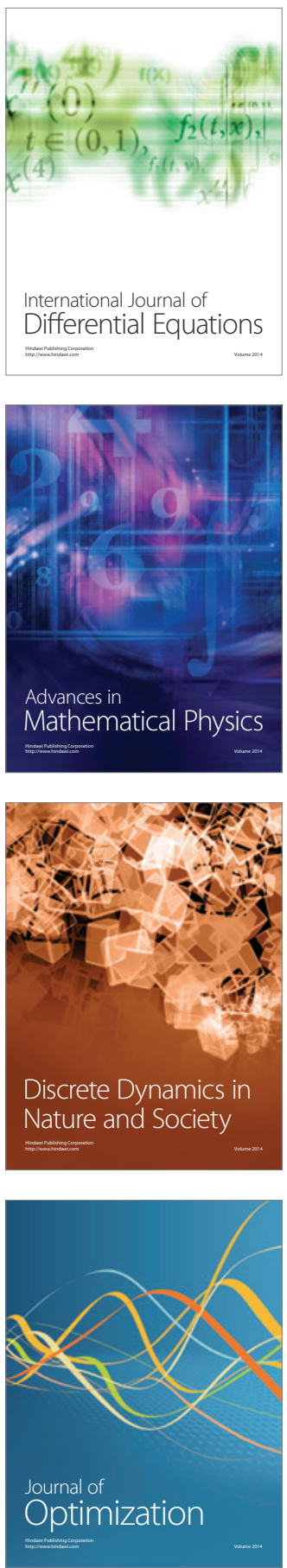